\title{
Knowledge, attitude and practice among non-ophthalmic health care providers regarding eye management of diabetics in private sector of Riyadh, Saudi Arabia
}

Talal Bassam Abu-Amara*, Waleed Abdulaziz Al Rashed, Rajiv Khandekar, Hamad Mohammed Qabha, Faris Mohammad Alosaimi, Abdulrahman Abdullah Alshuwayrikh, Mansour Khalid Almadi and Alanoud Alfaris

\begin{abstract}
Background: The levels of knowledge, attitude and practice among primary physicians concerning both diabetic retinopathy screening and treatment of sight threatening diabetic retinopathy have been studied by different groups, such as medical students, pharmacists, Primary Health Care staff and opticians. In some studies, the levels were very high, while in others it was noted to be less than desired.

Aim: This study's intent is to estimate and improve level of Knowledge (K), Attitude (A) and Practice (P) among non-ophthalmic health care providers regarding eye management of diabetes and barriers that people with diabetes face in Saudi Arabia.

Methods: This cross-sectional survey targeted medical doctors (except ophthalmologists) working at private sector institutions in Riyadh. They were interviewed using closed-ended questions for knowledge (8), attitude (5), practice (5), and reasons for their current KAP status comprised of 8 questions. The level of Knowledge was assessed as good if its score was (> 50\%); positive attitude (>50\%) and excellent practice (> 75\%) were estimated and associated to the risk factors.
\end{abstract}

Results: Out of the 355 participants that were interviewed, the percentages of good knowledge, positive attitude and excellent practice concerning diabetic retinopathy (DR)were 193 [54.3\% (95\% Cl 49.2-59.5)], 111 [31.3\% (95\% Cl 26.4-36.1)], and 145 [40.8\% (95\% Cl 35.7-46.0) participants, respectively. Gender, place of work and type of doctor were not significantly associated with the level of KAP. Salient reasons for low KAP status included a busy schedule $(54.6 \%)$, less resources $(75.2 \%)$, inadequate periodic training in eye care (69\%), and absence of retinal evaluation training (49.6\%).

Conclusions: Improving KAP level is urgently needed. Addressing underlying causes of low KAP could enhance eye care of people with diabetes. Additionally, training for primary health care providers for early detection of DR and timely management of sight threatening diabetic retinopathy (STDR) is necessary.

Keywords: KAP, Diabetes, Diabetic retinopathy, Primary health care providers, Screening

\footnotetext{
* Correspondence: talalabuamara@gmail.com

Department of Ophthalmology, Imam Mohammad Bin Saud Islamic

University, collage of medicine, Riyadh, Saudi Arabia
}

(c) The Author(s). 2019 Open Access This article is distributed under the terms of the Creative Commons Attribution 4.0 International License (http://creativecommons.org/licenses/by/4.0/), which permits unrestricted use, distribution, and reproduction in any medium, provided you give appropriate credit to the original author(s) and the source, provide a link to the Creative Commons license, and indicate if changes were made. The Creative Commons Public Domain Dedication waiver (http://creativecommons.org/publicdomain/zero/1.0/) applies to the data made available in this article, unless otherwise stated. 


\section{Background}

Physicians and family physicians are the first level health contacts for people with diabetes. Therefore, they are crucial for primary prevention-related counselling as well as proper guidance for further care by experts who deal with the systemic complications of diabetes [1]. In countries like Saudi Arabia, where both diabetes mellitus (DM) and diabetic retinopathy (DR) is of epidemic proportion [2,3], program approaches to deal with diabetes and diabetic retinopathy become of paramount importance [4]. Proper knowledge and the positive mind set of these health care providers could have a cascading effect in improving the practices both of caregivers and the adherence of people with diabetes to the diabetic care advice given to them [5]. Increasing private sector involvement in health care across the kingdom is evident. There were 1104 and 891 non-ophthalmic health care providers in private and governmental sector institutions of Riyadh respectively in 2016 [6].

Consequently, research about perspective of care providers should also focus on those working in the private sector due to their greater prevalence. The levels of knowledge, attitude and practice among Primary healthcare (PHC) physicians concerning both DR screening and treatment of sight-threatening diabetic retinopathy have been studied by different groups such as medical students [7], pharmacists [8], PHC staff [9] and opticians [10]. In some studies, the levels were very high while, in others, they were noted to be less than desired.

In addition to governmental institutions, the diabetic care in the study area is provided by both Saudi and non-Saudi health care providers in private institutions. Therefore, information of KAP of private sector primary physicians would complement about current status of non-ophthalmic care providers of people with diabetes to a large extent.

To the best of our knowledge KAP and barriers for eye care related to the knowledge and practice among private sector primary physicians have not been studied in central Saudi Arabia. Consequently, this study was conducted to assess the level of knowledge (K), attitude (A), and practice (P) among non-ophthalmic health care providers. Additionally, its intent was to improve the awareness of the early detection of DR and the timely management of sight-threatening diabetic retinopathy (STDR) in this group.

\section{Methods}

This cross-section type of study was undertaken between June and September 2017 and was approved by the Institution Review Board (IRB) of the $\mathrm{Al}$ Imam Mohammad Bin Saud Islamic University, Riyadh, Saudi Arabia [NO. 0014/12/016/31]. Doctors of randomly selected health institutions from the private sector, unrelated to secondary level eye care services, were invited to participate in this survey. Their verbal consent to participate in the survey was obtained.

We assumed that acceptable level of practice for DR screening was $53 \%$ in primary care physicians [9]. In the study area, there were 1100 non-ophthalmic primary health care professionals. The target was to achieve 95\% confidence interval with a 5\% acceptable error margin and 1.2 factor for clustering. The final sample size was comprised of 341 randomly selected professionals.

A health educator, five medical students and three ophthalmologists conducted this study. A pretested questionnaire was used to collect information regarding participant demographics, physician medical education and the doctors' interaction with patients during routine work in the work station. There were eight questions related to knowledge of DR diagnosis and management. The participants were given different options for response. If the reply of participants matched to the correct answer, per a panel of three ophthalmologist experts, 2 points were awarded. If the answer was incorrect, the participant received - 2 point. If a participant did not attempt to answer, 0 point were given. The sum of all responses for knowledge related question were further graded as: excellent, if the score was $>75 \%$ of the total possible score; good, if the score was between 51 and $75 \%$ and Poor, if the score was between 26 to $50 \%$. A participant having a score $25 \%$ or less was considered to have very poor knowledge of DR.

Next, there were five questions related to the attitude towards DR screening and its management. The responses were based on five graded Likert scale. The total score of the responses for attitude-related question were grouped as positive attitude if score was $>50 \%$ of the total correct responses, per the expert. If it was $<50 \%$, the attitude of the participant was considered negative.

Finally, there were five questions to inquire about the current practices for DR screening and DR management among participants while dealing with their patients. If the response of the participant matched to that of the expert panel, 2 points were allotted. If the answer was wrong, -2 points were awarded and for those who did not attempt the question, a 0 score was recorded. The total sum was further separated into excellent $(>75 \%$ score), good (50 to $75 \%$ ), poor (25 to $49 \%$ ) and very poor $(<25 \%)$.

The feedback of the participants was collected for improving the eye care of people with diabetes and the possible barriers that doctors face in improving their KAP regarding DR.

The data was collected using an English version of questionnaire. The responses were transferred into a spread sheet of Microsoft Excel ${ }^{\oplus}$. A univariate analysis, using the parametric method was carried out with the 
help of Statistical Package for Social Sciences (SPSS 24, IBM, Chicago, USA). The frequency and percentage proportions of the participants with excellent and good levels of knowledge, positive attitudes and acceptable (excellent + good) level of practice was associated to the demographic, medical education level and work-related variables. A two-sided $P$-value of $<0.05$ was considered statistically significant.

\section{Results}

\section{Demographic characteristics}

The present study recruited 355 non-ophthalmic health care providers. Four participants opted not to contribute to the survey after recruitment. The median age of the participants was 39 years-of-age $(25 \%$ quartile $=32$ yearsof-age) with a median of 13 years' experience after graduation $(25 \%$ quartile $=7$ years-of-experience) . Twothirds of the participants $(n=238,67 \%)$ were male, and the majority were non-Saudi $(n=352,99.2 \%)$. General practitioners constituted most of the enrolled physicians $(n=182,51.3 \%)$, followed by internists $(n=115,32.4 \%)$. More than two-thirds of the physicians were working in a polyclinic $(n=255,71.8 \%)$. Furthermore, one in ten physicians $(n=36)$ stated that they encountered diabetes in more than $50 \%$ of their patients. More demographic characteristics are given in Table 1.

\section{Knowledge}

Table 2 shows the eight knowledge-related questions and the participants' responses. The most incorrectly answered question was related to the changes that diabetes mellitus can cause in the eyes, with only $13.2 \%(n=47)$ answering it correctly. Conversely, the timing of screening a patient with newly diagnosed type 2 diabetes for DR was known to most of the participants $(n=279$, 78.6\%). The knowledge about DR screening and management was of an excellent level in $1.1 \%$ of participants $(n=4)$, good in $53.2 \%$ of participants $(n=189)$, and poor in $45.6 \%$ of participants $(n=162)$. Good + excellent knowledge was recorded in 193 participants [54.3\% (95\% CI 49.2-59.5)].

\section{The implication of demographic factors on KAP}

We correlated the levels of KAP with the demographic determinants in Table 3. Working in a hospital and male gender were found to be marginally related to a higher level of KAP for DR screening and management among the participants.

\section{Barriers to applying DR screening}

The participants' perceived barriers to applying DR screening are depicted in Table 4. A lack of resources ( $n=267,75.2 \%)$ followed by lack of adequate training
Table 1 Profile of participating non-ophthalmic health care providers in private sector of Riyadh

\begin{tabular}{|c|c|c|c|}
\hline \multirow[t]{2}{*}{ Age } & Median & \multicolumn{2}{|l|}{39.0} \\
\hline & $25 \%$ quartile & \multicolumn{2}{|l|}{32.0} \\
\hline \multirow[t]{2}{*}{ Years since graduated } & Median & \multicolumn{2}{|l|}{13} \\
\hline & $25 \%$ quartile & \multicolumn{2}{|l|}{7} \\
\hline \multirow[t]{2}{*}{ Years working in KSA } & Median & \multicolumn{2}{|l|}{6} \\
\hline & $25 \%$ quartile & \multicolumn{2}{|l|}{3} \\
\hline \multirow[t]{3}{*}{ OPD in a day } & Median & \multicolumn{2}{|l|}{22} \\
\hline & $25 \%$ quartile & \multicolumn{2}{|l|}{15} \\
\hline & & Number & Percentage \\
\hline \multirow[t]{2}{*}{ Gender } & Male & 238 & 67 \\
\hline & Female & 117 & 33 \\
\hline \multirow[t]{2}{*}{ Nationality } & Saudi & 3 & 0.8 \\
\hline & Non-Saudi & 352 & 99.2 \\
\hline \multirow[t]{9}{*}{ Type of care providers } & GPs & 182 & 51.3 \\
\hline & Internists & 115 & 32.4 \\
\hline & Family & 15 & 4.2 \\
\hline & Physicians & 15 & 4.2 \\
\hline & Endocrinologists & 17 & 4.8 \\
\hline & Surgeons & 3 & 0.8 \\
\hline & Paediatricians & 5 & 1.4 \\
\hline & Gynaecologists & 3 & 0.8 \\
\hline & Other & & \\
\hline \multirow[t]{2}{*}{ Work place } & Hospital & 100 & 28.2 \\
\hline & clinic & 255 & 71.8 \\
\hline \multirow[t]{5}{*}{ Category } & Consultant & 22 & 6.2 \\
\hline & Senior Specialist & 20 & 5.6 \\
\hline & Specialist & 119 & 33.5 \\
\hline & Resident & 23 & 6.5 \\
\hline & GP & 171 & 48.2 \\
\hline \multirow{3}{*}{$\begin{array}{l}\text { Proportion of diabetic } \\
\text { patient in OPD }\end{array}$} & less than $25 \%$ & 165 & 46.5 \\
\hline & 25 to $50 \%$ & 154 & 43.4 \\
\hline & More than $50 \%$ & 36 & 10.1 \\
\hline
\end{tabular}

( $n=245,69 \%)$ were the barriers most recognised by the participants.

Feedback regarding the capacity building of nonophthalmic eye care professionals in DR management suggested that $80.3 \%$ of participants $(n=285)$ believed that they needed hands-on training for DR screening, and $89.6 \%$ of participants $(n=318)$ suggested workshops on eye care in diabetes.

\section{Discussion}

The level of knowledge among non-ophthalmic physicians in the private sector concerning people with diabetes was less than desired, as nearly half of the 
Table 2 Knowledge related questions and responses of the participants

\begin{tabular}{|c|c|c|c|c|c|c|}
\hline \multirow[t]{2}{*}{ No } & \multirow[t]{2}{*}{ Questions } & \multicolumn{2}{|c|}{ Correct answers } & \multicolumn{2}{|c|}{ Wrong answers } & \multirow[t]{2}{*}{ The correct answers } \\
\hline & & Number & Percentage & Number & Percentage & \\
\hline 1 & $\begin{array}{l}\text { A } 50 \text {-year-old patient is first time diagnosed to suf- } \\
\text { fer from D.M type } 2 \text {, when should his retinal exam- } \\
\text { ination be done? }\end{array}$ & 279 & 78.6 & 76 & 21.4 & Soon after diagnosis \\
\hline 2 & $\begin{array}{l}\text { If your diabetes patient was told by eye doctor } \\
\text { that he/she does not have diabetic retinopathy. } \\
\text { When should be his/her next diabetic retinopathy } \\
\text { (DR) screening? }\end{array}$ & 242 & 68.2 & 113 & 31.8 & 1 year \\
\hline 3 & $\begin{array}{l}\text { A10 year-old child is diagnosed to have insulin } \\
\text { dependent D.M (Type 1). When should you send } \\
\text { him/her for diabetic retinopathy screening? }\end{array}$ & 119 & 33.5 & 236 & 66.5 & Within 3-5 years \\
\hline 4 & $\begin{array}{l}\text { How does a diabetes Patient usually describe lost } \\
\text { vision secondary to DR? }\end{array}$ & 275 & 77.5 & 80 & 22.5 & Gradual and painless \\
\hline 5 & $\begin{array}{l}\text { The risk of diabetic retinopathy is much higher and } \\
\text { more serious in diabetic patient of long duration in } \\
\text { which of the following complication of diabetes? }\end{array}$ & 160 & 45.1 & 195 & 54.9 & Diabetic nephropathy \\
\hline 6 & $\begin{array}{l}\text { Measures that can help in reducing the } \\
\text { progression of Diabetic Retinopathy }\end{array}$ & 102 & 28.7 & 253 & 71.3 & $\begin{array}{l}\text { Healthy lifestyle (regular exercise, good diet \& } \\
\text { stop smoking), Stringent blood lipid control } \\
\text { and Control of systemic blood pressure }\end{array}$ \\
\hline 7 & $\begin{array}{l}\text { Diabetes Mellitus can cause the following changes } \\
\text { in eye }\end{array}$ & 47 & 13.2 & 308 & 86.8 & $\begin{array}{l}\text { Macular edema, Cataract formation, Macular } \\
\text { ischemia andVitreous haemorrhage }\end{array}$ \\
\hline 8 & $\begin{array}{l}\text { Diabetes Mellitus can cause changes in eye which } \\
\text { of following changes require to emergency review } \\
\text { by ophthalmologist }\end{array}$ & 108 & 30.4 & 247 & 69.6 & $\begin{array}{l}\text { Sudden loss of vision, pre-retinal or vitreous } \\
\text { haemorrhage and Retinal detachment }\end{array}$ \\
\hline
\end{tabular}

participants had poor knowledge. The attitude for DR screening was found to be positive in one third of participants, which is a problem in that it may lead to the deterioration of the vision of patients with DR. The practice for preventive, by refereeing people with diabetes for early DR detection and following-up with patients after STDR management by an ophthalmologist, was promising in half of the participants (as shown in Table 5). The level of KAP of physicians of the private sector in our study varied according to their work place, which implicates the role of the institutions in the practice of doctors, as some have restrictions while others have good regulations. The participants felt an urgent need for training and creating a protocol for the early detection and management of DR, which is a good indicator for improvement.

A large number of people with DM need to be screened annually for DR in the Kingdom of Saudi Arabia to stop the progression of DR. In the view of ophthalmologists, one of the strategies to reach this goal is to shift the task of early detection to non-ophthalmic physicians [9]. This is possible only if the primary health care physicians are trained and have positive attitude concerning taking up this challenge [11]. Also, physicians of primary health care should counsel the patients regarding their cooperation for undergoing annual DR screening and the timely management of STDR, if the ophthalmologist recommends. Care for diabetes had been shown to improve when counselling is completed

Table 3 Factors related to good practices, positive attitude and good knowledge regarding diabetic retinopathy

\begin{tabular}{|c|c|c|c|c|c|c|c|c|c|c|}
\hline & & \multicolumn{3}{|c|}{ Knowledge } & \multicolumn{3}{|l|}{ Attitude } & \multicolumn{3}{|c|}{ Practice } \\
\hline & & Good & Poor & Validation & Positive & Not positive & Validation & Good & Poor & Validation \\
\hline \multirow[t]{2}{*}{ Gender } & Male & 125 & 113 & 0.3 & 78 & 159 & 0.4 & 95 & 141 & 0.55 \\
\hline & Female & 68 & 49 & & 33 & 82 & & 50 & 65 & \\
\hline \multirow[t]{2}{*}{ Place of work } & Clinics & 47 & 53 & 0.08 & 38 & 62 & 0.09 & 36 & 109 & 0.2 \\
\hline & Hospitals & 146 & 109 & & 73 & 182 & & 64 & 146 & \\
\hline \multirow[t]{4}{*}{ Type of specialists } & GPs + & 111 & 86 & 0.2 & 58 & 138 & 0.5 & 83 & 66 & 0.2 \\
\hline & Family & & & & & & & & & \\
\hline & Physicians & 78 & 80 & & 53 & 106 & & 103 & 107 & \\
\hline & Other & & & & & & & & & \\
\hline
\end{tabular}


Table 4 Barriers for DR screening as perceived by non-ophthalmic health care providers

\begin{tabular}{|c|c|c|c|c|c|c|c|}
\hline \multirow[t]{2}{*}{ No } & \multirow[t]{2}{*}{ Practice related Questions } & \multicolumn{2}{|l|}{ Yes } & \multicolumn{2}{|l|}{ No } & \multicolumn{2}{|l|}{ Not sure } \\
\hline & & Number & Percentage & Number & Percentage & Number & Percentage \\
\hline 1 & Busy in other health issues & 194 & 54.6 & 107 & 30.1 & 54 & 15.2 \\
\hline 2 & Lack of resources & 267 & 75.2 & 67 & 18.9 & 21 & 5.9 \\
\hline 3 & Policy for PHC conflict with mydriasis at PHCs & 170 & 47.9 & 97 & 27.3 & 88 & 24.8 \\
\hline 4 & Lack of adequate training & 245 & 69.0 & 78 & 22.0 & 32 & 9.0 \\
\hline 5 & Forgot how to examine retina & 176 & 49.6 & 133 & 37.5 & 46 & 13.0 \\
\hline 6 & Fear of precipitating glaucoma in patients & 158 & 44.5 & 120 & 33.8 & 77 & 21.7 \\
\hline 7 & Non-cooperation of diabetic patients & 123 & 34.6 & 112 & 31.5 & 120 & 33.8 \\
\hline 8 & Gender issue for DR screening & 154 & 43.4 & 100 & 28.2 & 101 & 28.5 \\
\hline
\end{tabular}

by the patients' attending doctors along with the management [12]. Hence, proper knowledge among physicians about diabetes eye complication and their relationship to visual disabilities is essential.

An excellent level of knowledge was documented in very few physicians in the present study. This was also noted in other studies of government institutions of Saudi Arabia [9], Oman [13], rural China [14] and South Africa [15]. Practitioners' busy schedules and non-sufficient continued medical education could be the causes for this lack of knowledge regarding DR [16]. One of the areas that can be improved upon is the continuous medical education to general practitioners, which has been demonstrated might improve the management of chronic non-communicable diseases, including diabetes [17]. Additionally, the present study's results showed that half of participants possessed good knowledge, however, regarding the practice of referrals, only $40 \%$ of patients with diabetes were referred for eye screening. The physicians of primary health care who were working in governmental sector of Riyadh had a knowledge score of $57 \% \pm 14$ and the correct practice of referring patients with Type I diabetic to the ophthalmologist was only $24 \%$ [9]. Thus, training and health promotion of health for non-ophthalmic physicians appears to be equally important for both private and governmental sector primary health care physicians.

As in the present study, there is also a weakness in knowledge concerning following up with patients with diabetes for DR screening and the relationship of diabetic duration with the development and progression of DR. However, this study was conducted with final year medical students in Saudi Arabia, which indicates a weakness in their basic clinical knowledge [7]. In another study, conducted in Oman, knowledge of the basic eye structure was satisfactory in only half of physicians, and the practice of fundus examination for DR was poor in $40 \%$ of physicians [13]. In practice, 20 physicians had attempted to use an ophthalmoscope, but only 9 could see the details of the retina; so, they also recommend detailed training for their physicians [13]. Moreover, a study was completed in China that stated that among 22 physicians, knowledge regarding eye complications in diabetes was generally good, but physicians did not favour routine pupillary dilation to detect asymptomatic disease; they expressed concerns about workflow as well as the danger and inconvenience to the patients [14]. Further, research from Cape Town, South Africa revealed deficiencies in training, resulting in consequent gaps in knowledge and practice regarding eye complications in diabetes [15].

Therefore, special focus is needed to improve knowledge about tele-screening for DR. A positive attitude towards eye care and laying down a protocol will ensure a uniform practice for DR screening and STDR management. Such recommendations have been periodically issued in Middle Eastern countries, as diabetes in Arabic-speaking countries is poorly controlled and the risk of diabetes complications in the eyes is high $[18,19]$.

Table 5 Practice related responses of non-ophthalmic health care professional for DR screening

\begin{tabular}{|c|c|c|c|c|c|c|c|c|}
\hline \multirow[t]{2}{*}{ No } & \multirow[t]{2}{*}{ Practice related Questions } & \multicolumn{2}{|l|}{ Good } & \multicolumn{2}{|c|}{ Not good } & \multicolumn{2}{|l|}{ Not sure } & \multirow{2}{*}{$\begin{array}{l}\text { The } \\
\text { correct } \\
\text { answers }\end{array}$} \\
\hline & & Number & Percentage & Number & Percentage & Number & Percentage & \\
\hline 1 & Mydriatic eye drops well maintained at Unit & 139 & 39.2 & 151 & 42.5 & 65 & 18.3 & Yes \\
\hline 2 & Ensure working condition of ophthalmoscope & 0 & 0.0 & 144 & 40.6 & 201 & 56.6 & Yes \\
\hline 3 & Refer all cases for DR screening & 161 & 45.4 & 22 & 6.2 & 10 & 2.8 & Yes \\
\hline 4 & Follows the referred diabetic to know DR status & 283 & 79.7 & 35 & 9.9 & 37 & 10.4 & Yes \\
\hline 5 & Follows patients treated for Dr by eye doctor & 307 & 86.5 & 27 & 7.6 & 21 & 5.9 & Yes \\
\hline
\end{tabular}




\section{Conclusions}

Improving KAP level is urgently needed. Addressing underlying causes of low KAP could enhance the eye care of patients with diabetes. Furthermore, training for primary health care providers regarding the early detection of DR and timely management of STDR is necessary.

\section{Abbreviations}

DM: Diabetes Mellitus; DR: Diabetic Retinopathy; IRB: Institution Review Board; KAP: Knowledge, Attitude, Practice; PHC: Primary healthcare; STDR: sight threatening diabetic retinopathy

\section{Acknowledgements}

Not applicable.

\section{Authors' contributions}

TBA, WAA and RK participated in study design. WAA, TBA, FMA, AAA, HMQ, $M K A, R K$, and $A A$ designed the study questionnaire and drafted the manuscript. TBA, FMA, AAA, MKA, and $\mathrm{HMQ}$ were involved in data collection. RK contributed to data analysis. WAA supported and supervised study implementation. TBA reviewed the final manuscript. All authors read and approved the final manuscript.

\section{Funding}

This research was not funded.

\section{Availability of data and materials}

The datasets used for the current study are not publicly accessible due to ethical reasons but are available from the corresponding author on reasonable request.

\section{Ethics approval and consent to participate}

The study was ethically approved by the Institutional Review Board (IRB) of Al Imam Mohammad Bin Saud Islamic University, Riyadh, Saudi Arabia [Number. 0014/12/016/31]. All the participants provided their verbal consent before participating in the study and the ethics committee agreed with this method of collecting consent form participants because it was easier to explain to participants about the idea of research and it is faster because we were dealing with physicians.

\section{Consent for publication}

Not applicable.

\section{Competing interests}

The authors declare that they have no competing interests.

Received: 19 August 2018 Accepted: 4 June 2019

Published online: 13 June 2019

\section{References}

1. Chamberlain JJ, Rhinehart AS, Shaefer CF, Neuman A. Diagnosis and Management of Diabetes: synopsis of the 2016 American Diabetes Association standards of medical care in DiabetesSynopsis of the 2016 ADA standards of medical Care in Diabetes. Ann Intern Med. 2016;164(8):542-52.

2. Moradi-Lakeh M, Forouzanfar MH, El Bcheraoui C, Daoud F, Afshin A Hanson SW, et al. High fasting plasma glucose, Diabetes,and its risk factors in the eastern Mediterranean region, 1990-2013: FindingsFrom the global burden of disease study 2013. Diabetes Care. 2017;40(1):22-9.

3. Alaboud AF, Tourkmani AM, Alharbi TJ, Alobikan AH, Abdelhay O, Al Batal SM, et al. Microvascular and macrovascular complications of type 2diabetic mellitus in central, Kingdom of Saudi Arabia. Saudi Med J. 2016:37(12):1408-11

4. Murthy GV, Gilbert CE, Shukla R, Vashist P, Shamanna BR. Situational analysis of services for diabetes and diabetic retinopathy and evaluation of programs for the detection and treatment of diabetic retinopathy in India: methods for the India 11-city 9-state study. Indian J Endocrinol Metabol. 2016;20(Suppl 1):S19.
5. Larkin AT, Hoffman C, Stevens A, Douglas A, Bloomgarden Z. Determinants ofadherence to diabetes treatment. J Diabetes. 2015;7(6):864-71.

6. Ministry of Health. Kingdom of Saudi Arabia. Health Resources in Statistical Year Book 2016. Table 2-17. Physicians And Dentists, Moh Hospitals By Speciality, Grade And Region. P-63. https://www.moh.gov.sa/en/Ministry/ Statistics/book/Documents/StatisticalBook-1436.pdf. Accessed 15 Feb 2018.

7. Al Wadaani FA. The knowledge attitude and practice regarding diabetes anddiabetic retinopathy among the final year medical students of king FaisalUniversity medical College of Al Hasa region of Saudi Arabia: a cross sectionalsurvey. Niger J ClinPract. 2013;16(2):164-8.

8. El Hajj MS, Abu Yousef SE, Basri MA. Diabetes care in Qatar: a survey of pharmacists' activities, attitudes and knowledge. Int J Clin Pharm. 2018;40(1): 84-93.

9. Al Rasheed R, Al Adel F. Diabetic retinopathy: knowledge, awareness andpractices of physicians in primary-care centers in Riyadh, Saudi Arabia. Saudi J Ophthalmol. 2017;31(1):2-6.

10. Wright SE, McKay R, Taylor Kl, et al. Changes in attitudes and practices of optometrists in their management of diabetic retinopathy after the release of NHMRC guidelines. National Health Med Res Council Clin Exp Ophthalmol. 2001;29(3):121-4.

11. Micheletti JM, Hendrick AM, Khan FN, Ziemer DC, Pasquel FJ. Current and NextGeneration portable screening devices for diabetic retinopathy. J Diabetes SciTechnol. 2016;10(2):295-300

12. Daskivich LP, Vasquez C, Martinez C Jr, Tseng CH, Mangione CM. Implementation and evaluation of a large-scale Teleretinal diabetic retinopathy ScreeningProgram in the Los Angeles County Department of Health Services. JAMA Intern Med. 2017;177(5):642-9.

13. Khandekar R, Shah S, Al Lawatti J. Retinal examination of diabetic patients: knowledge, attitudes and practices of physicians in Oman. East Mediterr Health J. 2008;14(4):850-7.

14. Yan X, Liu T, Gruber L, He M, Congdon N. Attitudes of physicians, patients, and village health workers toward glaucoma and diabetic retinopathy in rural China: a focus group study. Arch Ophthalmol. 2012;130(6):761-70.

15. Goodman GR, Zwarenstein MF, Robinson II, Levitt NS. Staff knowledge, attitudesand practices in public sector primary care of diabetes in Cape Town. S Afr MedJ. 1997;87(3):305-9.

16. Brown JB, Harris SB, Webster-Bogaert S, Wetmore S, Faulds C, et al. The role of patient, physician and systemic factors in the management of type 2 diabetes mellitus. Fam Pract. 2002;19(4):344-9.

17. Katulanda P, Constantine GR, Weerakkody MI, Perera YS, Jayawardena MG, Wijegoonawardena $\mathrm{P}$, et al. Can we bridge the gap? Knowledge andpractices related to diabetes mellitus among general practitioners in adeveloping country: a cross sectional study. Asia Pac Fam Med. 2011;10(1):15.

18. Khandekar R. Screening and public health strategies for diabetic retinopathyin the eastern Mediterranean region. Middle East Afr J Ophthalmol. 2012;19(2):178-84

19. Badran M, Laher I. Type II diabetes mellitus in Arabic-speaking countries. IntJ Endocrinol. 2012;2012:902873.
Ready to submit your research? Choose BMC and benefit from:

- fast, convenient online submission

- thorough peer review by experienced researchers in your field

- rapid publication on acceptance

- support for research data, including large and complex data types

- gold Open Access which fosters wider collaboration and increased citations

- maximum visibility for your research: over $100 \mathrm{M}$ website views per year

At $\mathrm{BMC}$, research is always in progress.

Learn more biomedcentral.com/submissions 